\title{
Phytochemical Isoliquiritigenin Inhibits Angiogenesis Ex Vivo and Corneal Neovascularization in Mice
}

\author{
Lingling Yang ${ }^{1}$, Wenxiao He ${ }^{1}$, Huihua Qu ${ }^{2}$, Changkai Jia ${ }^{1}$, Yao Wang ${ }^{1}$, Yiqiang Wang ${ }^{1,3^{*}}$ and Dongmin Liu ${ }^{2^{*}}$ \\ ${ }^{1}$ Shandong Provincial Key Lab of Ophthalmology, Shandong Eye Institute, Shandong Academy of Medical Sciences, Qingdao, China \\ ${ }^{2}$ Department of Human Nutrition, Foods and Exercise, Virginia Tech, Blacksburg, USA \\ ${ }^{3}$ University Research Center, Beijing University of Traditional Chinese Medicine, Beijing, China
}

*Corresponding author: Dongmin Liu, Department of Human Nutrition, Foods and Exercise, Virginia Tech, USA, Tel: 540/231-3402; Fax: 540/231-3916; Email: doliu@vt.edu

Rec date: Aug 04, 2014; Acc date: Nov 19, 2014; Pub date: Nov 21, 2014

Copyright: @ 2014 Liu D, et al. This is an open-access article distributed under the terms of the Creative Commons Attribution License, which permits unrestricted use, distribution, and reproduction in any medium, provided the original author and source are credited.

\begin{abstract}
Neovascularization is often involved in many diseases and there is no effective treatment for this pathological process. In searching for potential therapies for neovascularization, we screened nineteen pre-selected small molecules isolated from herbal extracts for their possible anti-angiogenic effect in vitro and in vivo. We found that isoliquiritigenin, a chalconoid compound isolated from Chinese herb medicine licorice, potently inhibited vascular endothelial cell (EC) proliferation, migration, tube -like structure formation ex vivo. Western blot analysis shows that exposure of ECs to isoliquiritigenin inhibited extracellular signal-regulated kinase 1/2 (ERK1/2) phosphorylation. In Matrigel plug assay, isoliquiritigenin effectively blocked fibroblast growth factor-induced in vivo angiogenesis in mice. Consistently, topical application of isoliquiritigenin significantly inhibited chemical injury-induced corneal neovascularization in mice. Collectively, these results suggest that isoliquiritigenin may be a low-cost and effective natural agent to treat angiogenesis-dependent diseases.
\end{abstract}

Keywords: Isoliquiritigenin; Vascular endothelial cell; Proliferation; Migration; Angiogenesis; Neovascularization

\section{Introduction}

Angiogenesis is a complex phenomenon long known to be under the tight control by a large panel of cytokines, cells, hormones, etc. [1]. While angiogenesis might occur under both physiological and pathological conditions, neovascularization is often referred to the development of new blood vessels in tissues that are normally not or less vascularized. Except for situations where neovascularization helps to heal and reconstitute the structure of injured tissues, all other instances of neovascularization are associated with the dysfunction of the affected tissues or with the expansion of undesirable tissues (e.g. tumor) [2]. The cornea is normally avascular. Under pathological conditions however, blood vessels from the limbal vascular plexus could invade the cornea, leading to its pathological vascularization that can cause visual impairment and blindness [3,4]. Previous studies showed that corneal neovascularization can be triggered by various factors, including infection [5], trauma [6] and inflammation [7]. While several strategies have been tested for their antineovascularization efficacy, such as multiple anti-angiogenic gene therapy [8], recombinant anti-angiogenic factor therapy [9], and antiinflammation therapy with monoclonal antibody against proinflammatory molecules [10], these approaches are not very effective in the treatment of corneal neovascularization [11].

Natural products (e.g. medicinal herbs) have been proposed as alternative resources for anti-angiogenic agents [8,12]. Indeed, traditional Chinese Medicine practitioners have been prescribing herbal medicines to treat various diseases that are associated with neovascularization, although not all of herbal therapies are based on evidence from the results of modern biomedical studies [13]. In addition, the components that are responsible for the anti-angiogenic action of these herb medicines are largely unknown. In the present study, we first screened 19 small molecules isolated from presumably angiogenesis-modulating herbs for their potential to modulate endothelial cell (EC) behavior ex vitro. We then studied the antiangiogenic effect of the identified compound in vivo and further determined the mechanism underlying this action. We found that isoliquiritigenin, a chalconoid compound isolated from Licorice Roots, is a potent agent for the treatment of pathological neovascularization.

\section{Materials and Methods}

\section{Herbal isolates}

All compounds were isolated and provided by the National Pharmaceutical Engineering Center for Chinese Herbal Medicine in Jiangxi Herbfine Hi-tech Co., Ltd, Nanchang, Jiangxi, China. The purity of all compounds is $>98 \%$ as determined by high performance liquid chromatography. The compounds were dissolved, either in PBS or dimethyl sulfoxide (DMSO), according to the manufacturer's instructions.

\section{Cell proliferation assay}

Primary human umbilical vein endothelial cells (HUVECs) were used for the proliferation analyses. Cell proliferation was measured using the coloregenic Cell Counting Kit-8 (CCK, Dojindo laboratories, Kumamoto, Japan) as previously described [14]. In brief, HUVECs were grown to about $30 \%$ confluence followed by the addition of the individual compounds or vehicle at a single concentration of $40 \mu \mathrm{M}$. After $48 \mathrm{~h}$ of incubation, CCK- 8 solution was added and the cells were 
incubated for additional $4 \mathrm{~h}$. The absorbance at $450 \mathrm{~nm}$ was measured using a microplate reader (Molecular Devices, Sunnyvale, CA, USA).

\section{Cell migration assay}

Migration of HUVECs was evaluated by using a monolayer wound healing and the Transwell migration assays. For the wound healing assay, cells were plated on 24-well culture plates and allowed to grow to confluence. A scratch was made with a micropipette tip and the cultures were rinsed to remove detached cells. Cells were then cultured in medium with or without various concentrations of tanshinone IIA or isoliquiritigenin. On the outer surface of the culture wells facing the scratches, ink lines were made perpendicular to the inside scratch and the width of the scratches at the crossing site were photographed before and after the $24 \mathrm{~h}$ of incubation. The cell migration rates were calculated as previously described [15].

For Transwell migration assay, HUVECs were placed in the upper compartment of Transwell Boyden chambers with fibronectin-coated polycarbonate filters (8- $\mu \mathrm{m}$ pore size, Corning Inc., Acton, MA) at $1 \times 105$ cells/well in $0.2 \mathrm{~mL}$ medium containing $1 \%$ serum. The lower chamber contained $0.7 \mathrm{~mL}$ of complete medium supplemented with increasing concentrations of isoliquiritigenin $(0,25,50$, or $100 \mu \mathrm{M})$. After $6 \mathrm{~h}$ of incubation at $37^{\circ} \mathrm{C}$, cells remaining on the upper surface of filter were removed using a cotton swab. The cells that had transmigrated to the lower surface of the membranes were stained with $0.1 \%$ crystal violet and counted under microscope.

\section{In vitro angiogenesis assay}

Thirty $\mu \mathrm{L}$ of Matrigel ${ }^{\mathrm{m}}$ Matrix (BD Bioscience, San Jose, CA) was dispensed in 96-well plates and allowed to polymerize for $1 \mathrm{~h}$ at $37^{\circ} \mathrm{C}$. HUVECs were labeled with Vybrant Dil cell-labeling solution (Invitrogen, Carlsbad, CA, USA) and seeded at a density of $1.5 \times 104$ cells/well in medium containing various concentrations of isoliquiritigenin $(0,6.25,12.5,25,50$ or $100 \mu \mathrm{M})$. Cells were then incubated at $37^{\circ} \mathrm{C} .6 \mathrm{~h}$ later, random fields were photographed using an Eclipse TE2000-U microscope (Nikon, Japan) with a $594 \mathrm{~nm}$ filter.

\section{Western blot analysis}

Protein extracts from treated and untreated HUVECs were resolved on $10 \%$ SDS-PAGE gel and then transferred to nitrocellulose membranes. The membranes were probed with antibody against phosphorylated extracellular signal-regulated kinase 1/2 (ERK1/2; Santa Cruz Biotechnology, Santa Cruz, CA) according to the manufacturer's instructions and the immunoreactive proteins were detected by chemilluminescence. The membranes were then stripped and re-probed with ERK 1/2 antibody (Santa Cruz).

\section{In vivo Matrigel plug angiogenesis assay}

C57BL/6 mice (6-10 weeks old) were used for in vivo assays of angiogenesis. Mice were purchased from the Laboratory Animal Center of the Academy of Military Medical Sciences (Beijing, China) and all experimental procedures were carried out in accordance with the guidelines of the Association for Research in Vision and Ophthalmology Statement for the Use of Animals in Ophthalmic and Vision Research. To assess the effect of isoliquiritigenin on angiogenesis in vivo, $300 \mu \mathrm{l}$ of Matrigel (BD Bioscience, San Jose, CA, USA) containing $60 \mathrm{ng}$ of fibroblast growth factor (R\&D System, Minneapolis, MN, USA) and 3U of heparin (B.Braun Melsungen AG,
Melsungen, Germany), with or without $100 \mu \mathrm{M}$ of isoliquiritigenin, were injected subcutaneously into the abdominal wall of mice $(n=4$ mice/group). Ten days after implantation, mice were euthanized and the Matrigel plugs were removed and photographed. Hemoglobin contents in the plugs were measured as described by others [16] with modification. Briefly, the implants were snap frozen, lyophilized overnight, and weighted. The dried Matrigel was re-suspended in 0.4 $\mathrm{mL}$ of $0.1 \%$ Triton $\mathrm{X}-100$ for $1 \mathrm{~h}$, disrupted by vigorous pipetting, and then centrifuged at $14,000 \times \mathrm{g}$ for $15 \mathrm{~min}$ to remove any particulate debris. Absorbance of hemoglobin in the supernatants was read at 405 $\mathrm{nm}$ with a microplate reader, and its concentration was calculated according to the standard curve obtained with pure hemoglobin.

\section{Corneal neovascularization studies}

Under general anesthesia with intraperitoneal injection of ketamine and chloropromazine $(50 \mathrm{mg} / \mathrm{kg}$ and $5 \mathrm{mg} / \mathrm{kg}$, respectively), corneal neovascularization in mice were generated by application of a $2 \mathrm{~mm}$ sized filter soaked with $2 \mu \mathrm{L}$ of $1 \mathrm{~N} \mathrm{NaOH}$ to the central cornea for 40 sec followed by immediate rinsing with normal saline for $40 \mathrm{sec}$, a widely used mouse model for pathological corneal neovascularization. Isoliquiritigenin or resveratrol $(100 \mu \mathrm{M}$ in saline eye drops) was applied topically $(5 \mu \mathrm{L})$ to the burned eyes 4 times daily for 7 consecutive days. Control eyes were similarly burnt but treated with saline only. At day 7, eyes were photographed under a slit lamp. All mice were killed and their corneas were collected and subjected to conventional histology with hematoxylin-eosin (H\&E) staining and immunohistochemistry as detailed below.

\section{Histology and immunohistochemistry}

Eyeballs were either fixed with formalin or snap-frozen in Optimal Cutting Temperature (OCT) compound (Sakura Finetechnical, Tokyo, Japan). Formalin-fixed tissues were stained with H\&E. For immunofluorescence staining, cryosections $(6 \mu \mathrm{m})$ were prepared from OCT-embedded eyeballs and were fixed in ice-cold acetone for $10 \mathrm{~min}$. The sections were blocked with $10 \%$ normal goat serum for 15 min and then stained with PE-conjugated anti-CD31 monoclonal antibody (1:100, BD Biosciences, San Jose, CA, USA) overnight at $4^{\circ} \mathrm{C}$. After counterstaining with DAPI, the stained sections were viewed under an Eclipse TE2000-U microscope (Nikon, Japan). Negative controls were stained in parallel by omitting primary antibody.

\section{Reverse-transcription quantitative real-time PCR analysis}

Total RNA from mouse corneas was extracted using a NucleoSpin RNAII kit (Macherey-Nagel, Düren, Germany) and reverse transcribed using a PrimeScript RT Reagent Kit (Takara, BIO INC., Shiga, Japan) following the manufacturer's protoocls. Quantitative PCR analysis of vascular endothelial growth factor (VEGF), basic fibroblast growth factor (bFGF), and transforming growth factor- $\beta 1$ (TGF- $\beta 1$ ) was performed in an ABI 7500 Detection System (Applied Biosystems, Foster City, CA, USA) using TaqMan Mastermix (Tiangen Biotech, Beijing China). RPL5 gene was used as internal control. Reactions were carried out with an initial 10 -min incubation at $95^{\circ} \mathrm{C}$ followed by 45 cycles at $95^{\circ} \mathrm{C}$ for $15 \mathrm{sec}$ and then at $60^{\circ} \mathrm{C}$ for $1 \mathrm{~min}$. The raw data were analyzed using SDS 7500 software (Applied Biosystems) and $\mathrm{Ct}$ values for each gene in each sample were determined. The relative amount of each target gene mRNA to RPL5 was calculated as the 2- $\Delta \mathrm{Ct}$ (where $\Delta \mathrm{Ct}=\mathrm{Ct}-\mathrm{CtRPL5}$ ) and expressed as folds of control. The primers used in quantitative real-time RT-PCR were listed below (Table 1): 


\begin{tabular}{|l|l|l|}
\hline $\begin{array}{l}\text { Gene Symbol } \\
\text { (Accession } \\
\text { Number) }\end{array}$ & $\begin{array}{l}\text { Primer sequences } \\
\text { F: forward; R:reverse }\end{array}$ & $\begin{array}{l}\text { Amplico } \\
\mathbf{n} \\
\text { (bp) }\end{array}$ \\
\hline $\begin{array}{l}\text { VEGF } \\
\text { (NM_009505) }\end{array}$ & $\begin{array}{l}\text { F- GCTACTGCCGTCCGATTGAG } \\
\text { R- CACACAGGACGGCTTGAAGA }\end{array}$ & $86 \mathrm{bp}$ \\
\hline $\begin{array}{l}\text { bFGF2 } \\
\text { (NM_008006) }\end{array}$ & $\begin{array}{l}\text { F-AGAGCGACCCACACGTCAA } \\
\text { R-AAGGTACCGGTTGGCACACA }\end{array}$ & $86 \mathrm{bp}$ \\
\hline $\begin{array}{l}\text { TGFB1 } \\
\text { (NM_011577) }\end{array}$ & $\begin{array}{l}\text { F-ACGGAATACAGGGCTTTCGA } \\
\text { R-GCTGATCCCGTTGATTTCCA }\end{array}$ & $86 \mathrm{bp}$ \\
\hline $\begin{array}{l}\text { RPL5 } \\
\text { (NM_016980) }\end{array}$ & $\begin{array}{l}\text { F- GGAAGCACATCATGGGTCAGA } \\
\text { R-TACGCATCTTCATCTTCCTCCATT }\end{array}$ & $70 \mathrm{bp}$ \\
\hline
\end{tabular}

Table 1: Sequences of primers used for RT-qPCR assay.

\section{Statistical analysis}

Data from in vitro experiments were derived from at least three independent experiments with duplicated determination each. Variation within treatments is expressed as the standard error (SE) of the mean (SEM) for in vitro assays and SE for in vivo studies. All data were subjected to a one-way ANOVA using the SigmaPlot software and treatment differences were subjected to Tukey's multiple comparison tests, where $\mathrm{P}<0.05$ was considered significant.

\section{Results}

\section{Effects of herb-derived small molecules on EC proliferation}

For this study, 19 pure compounds were carefully selected based on the following reasons. First, the herbs from which these molecules were isolated have been used in complex recipes of traditional Chinese Medicine for treating various angiogenesis-related diseases; and second, these selected chemicals had been thought to be bioactive components responsible for some biological effects of corresponding herb(s). These 19 molecules are arteannuinum succinate, baicalein, berberine hydrochloride, ginkgolide $\mathrm{B}$, ginsenoside $\mathrm{Rg} 3$, hydroxycamptothecin, isoliquiritigenin, L-epicatechin, ligustrazine hydrochloride, notoginsenoside $\mathrm{R} 1$, procyanidin $\mathrm{B} 2$, protocatechuic aldehyde, puerarin, quercetin, resveratrol, salvianic acid A sodium, silybin, tanshinone IIA, and wogonin. Beside we first tested their effects on the proliferation of primary human umbilical vascular ECs (HUVECs). In that regard, cells were cultured in the medium with the presence of vehicle or $40 \mu \mathrm{M}$ various isolated compounds for $24 \mathrm{~h}$. The results showed that baicalein, tanshinone II A, resveratrol, ginsenoside Rg3, arteannuinum succinate, berberine hydrochloride, isoliquiritigenin, and 10-hydroxy camptothecin inhibited cell proliferation, whereas ligustrazine hydrochloride, pueraria, notoginsenoside R1, epicatechin, and silybin stimulated cell proliferation as compared to the control (Figure 1). The order of potency for the inhibition of EC proliferation at this concentration is tanshinone

IIA $\approx$ isoliquiritigenin $>$ arteannuinumsuccinate $\approx$ berberinehydrochlorid e $>$ hydroxycamptothecin $>$ ginsenoside $\operatorname{Rg} 3>$ resveratrol $\approx$ Baicalein, with tanshinone IIA and isoliquiritigenin blocking cell proliferation by $97.0 \%$ and $93.7 \%$, respectively. We further tested does-dependent effects of tanshinone IIA and isoliquiritigenin on EC proliferation. Both tanshinone IIA (Figure 2A) and isoliquiritigenin (Figure 2B) dose-dependently inhibited EC proliferation. Tanshinone IIA as low as $5 \mu \mathrm{M}$ significantly inhibited EC proliferation with $10 \mu \mathrm{M}$ and $20 \mu \mathrm{M}$ concentrations blocking cell proliferation by $64 \%$ and $94 \%$, respectively, whereas isoliquiritigenin at $\leq 10 \mu \mathrm{M}$ concentrations was ineffective with $10 \mu \mathrm{M}$ reducing proliferation by $42 \%$, suggesting that low-dose tanshinone IIA is more potent than isoliquiritigenin in inhibiting EC proliferation. To confirm that the effect of Tanshinone IIA and isoliquiritigenin in our experiments was on cell proliferation, rather than due to induction of cell death, we assessed if tanshinone IIA and isoliquiritigenin induce apoptosis of HUVECs. In that regard, cells were treated with various doses of tanshinone IIA or isoliquiritigenin for $24 \mathrm{~h}$. The results showed that tanshinone IIA or isoliquiritigenin ISL at $\leq 80 \mu \mathrm{M}$ had no effect on cell apoptosis (data not shown). Since the long-term purpose of this study is to identify herb-derived small molecules that will be potentially used in managing human neovascularization diseases, we focused following studies on these two most potent compounds that inhibited HUVEC proliferation.

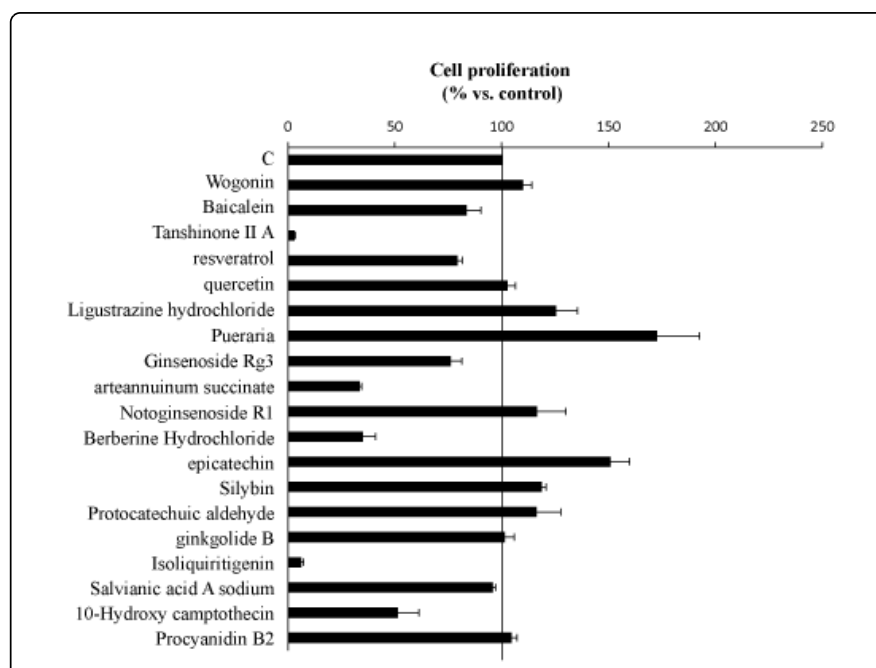

Figure 1: The effect of herb-derived compounds on EC proliferation. HUVECs were incubated with vehicle or $40 \mu \mathrm{M}$ individual compounds. After $48 \mathrm{~h}$ of incubation, cell proliferation was measured as described in Methods. Data are expressed as mean \pm SEM of three separate experiments with quadruplicate determinations each. ${ }^{*} \mathrm{P}<0.05$ vs vehicle alone-treated cells.

\section{Effects of tanshinone IIA and isoliquiritigenin on EC migration}

The chemotactic migration of ECs is one of the critical steps in the process of angiogenesis. We therefore examined whether tanshinone IIA and isoliquiritigenin can further inhibit migration of HUVECs. We first performed the monolayer wound healing assay to evaluate whether these compounds affect EC migration. The results showed that both tanshinone IIA and isoliquiritigenin dose-dependently inhibited cell migration from the edge of the wound into the open area, but isoliquiritigenin is more potent than Tanshinone IIA in inhibiting the migration of HUVECs (Figure $3 \mathrm{~A}$ and $3 \mathrm{~B}$ ). Next, we performed transwell assays by using modified Boyden chambers to further evaluate whether isoliquiritigenin affects cell migration in HUVECs. As shown in Figure 3C, isoliquiritigenin significantly 
Citation: Yang L, He W, Qu H, Jia C, Wang Y, et al. (2014) Phytochemical Isoliquiritigenin Inhibits Angiogenesis Ex Vivo and Corneal Neovascularization in Mice. Altern Integr Med 3: 176. doi:10.4172/2327-5162.1000176

reduced the number of migrated cells through the membrane barrier of the transwell.

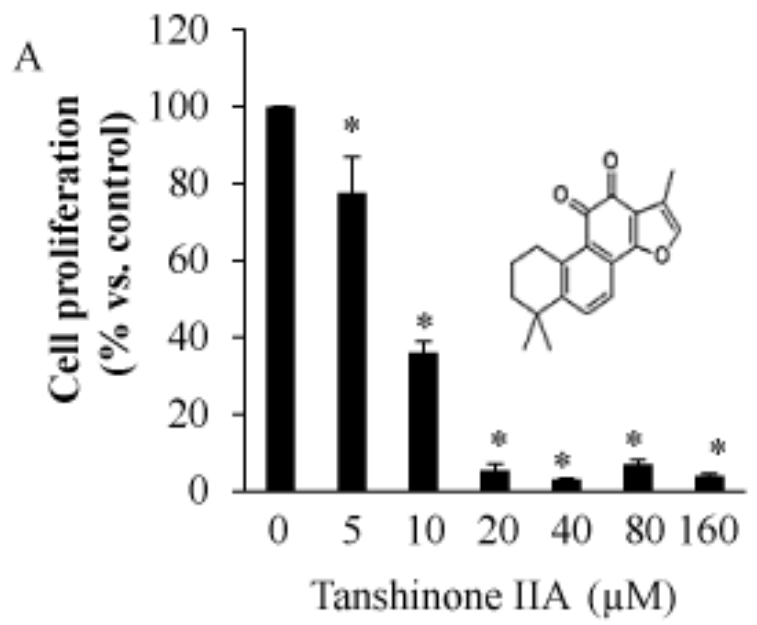

B

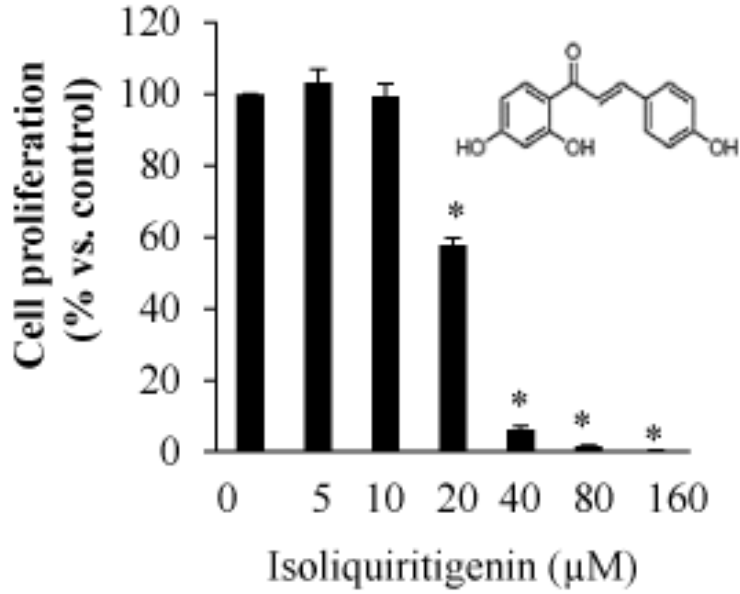

Figure 2: Does-dependent effects of Tanshinone IIA and isoliquiritigenin on EC proliferation. HUVECs were incubated with vehicle or various concentrations of Tanshinone IIA (A) or isoliquiritigenin (B) After $48 \mathrm{~h}$ of incubation, cell proliferation was measured as described in Methods. Data are expressed as mean \pm SEM of three separate experiments with quadruplicate determinations each. ${ }^{*} \mathrm{P}<0.05$ vs vehicle alone-treated cells.

\section{Isoliquiritigenin abolishes in vitro angiogenesis}

Angiogenesis is a complex process involving EC proliferation, migration, and the formation of vascular tube structures. Next, we evaluated if isoliquiritigenin affects EC tube structure formation, a widely used in vitro model of angiogenesis. In that regard, cells were plated onto Matrigel, followed by incubation with or without isoliquiritigenin. As shown in Figure 4, ECs developed tube/cord-like networks after $6 \mathrm{~h}$ of incubation. However, exposure of cells to isoliquiritigenin substantially ablated tube-like structure formation.
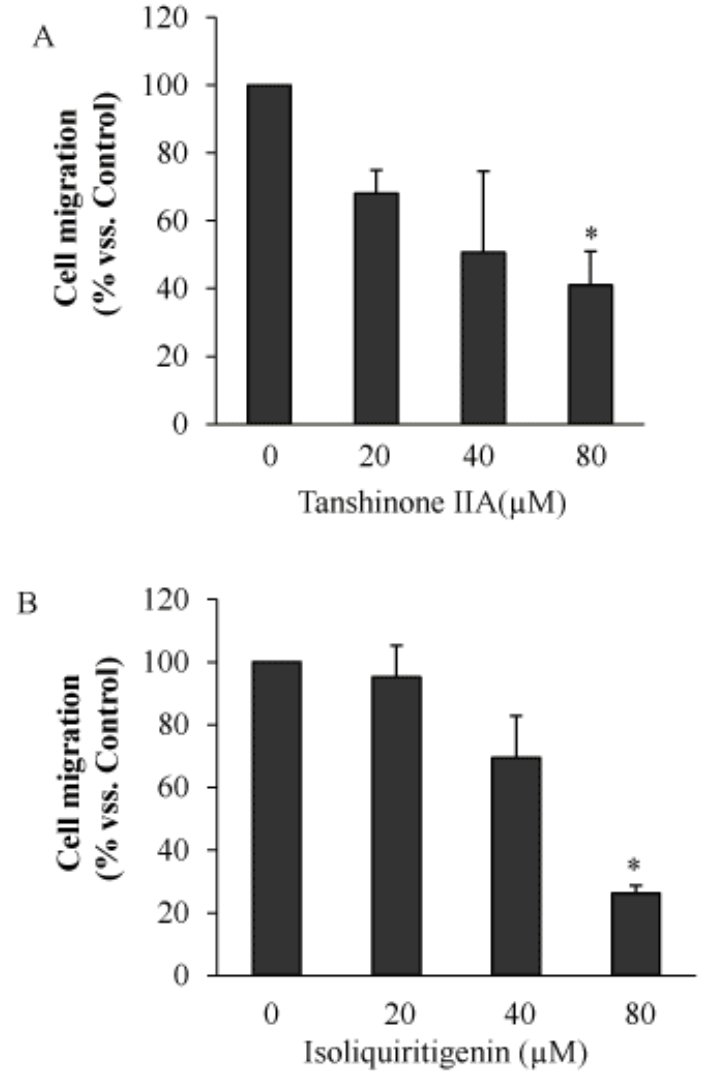

C

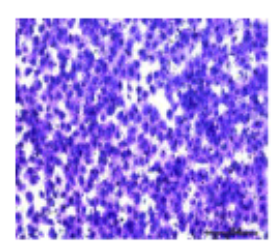

0

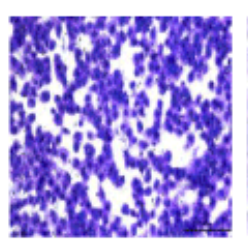

40

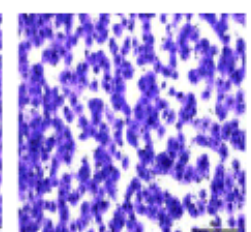

80

Isoliquiritigenin $(\mu \mathrm{M})$

Figure 3: The effects of Tanshinone IIA and isoliquiritigenin on migration of HUVECs. Cell cultures were "wounded" and incubated in medium with or without various concentrations of Tanshinone IIA (A) or isoliquiritigenin (B) for $24 \mathrm{~h}$. The distance between the two wound edges was measured before and after treatment and are expressed as a percentage of the control. Representative photographs are shown of cells immediately after wound induction or after $24 \mathrm{~h}$ incubations with or without compounds from three independent experiments. Cell migration was determined by modified Boyden chamber assay in the presence of isoliquiritigenin or vehicle (C), as described in Methods. Data are expressed as mean \pm SEM of three separate experiments with quadruplicate determinations each. Inserts in bar graphs are chemical structures. ${ }^{*} \mathrm{p}<0.05$ vs. control. 
Citation: Yang L, He W, Qu H, Jia C, Wang Y, et al. (2014) Phytochemical Isoliquiritigenin Inhibits Angiogenesis Ex Vivo and Corneal Neovascularization in Mice. Altern Integr Med 3: 176. doi:10.4172/2327-5162.1000176

Page 5 of 8

\section{Isoliquiritigenin may inhibit angiogenesis through suppression of ERK1/2 activity}

It is well recognized that the ERK $1 / 2$ signaling pathway plays an important role in cell proliferation and growth. Next, we investigated the possible pathways involved in the modulation of angiogenesis by comparing the total and activated ERK1/2 levels in isoliquiritigenintreated and untreated HUVECs. The results from Western blot analysis showed that isoliquiritigenin induced a dose-dependent inhibition of the phosphorylation of ERK1/2, a magnitude similar to its effect on cell proliferation and migration (Figure 5). However, isoliquiritigenin had no effect on total ERK protein expression in the cells.

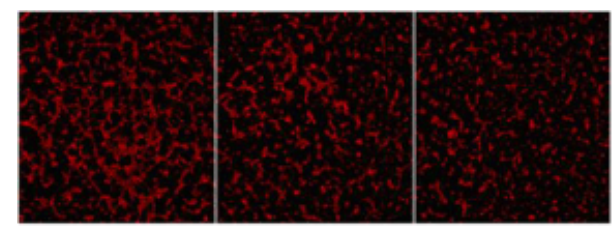

$$
\begin{array}{c|c|c}
0 & 6.25 & 12.5 \\
\hline 25 & 50 & 100 \\
\text { Isoliquiritigenin }(\mu \mathrm{M})
\end{array}
$$

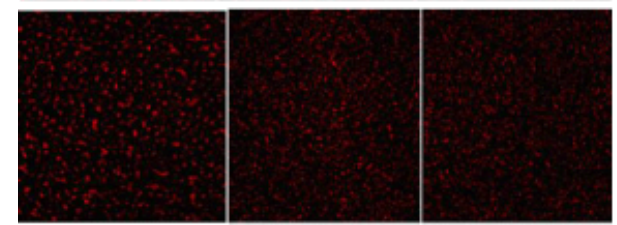

Figure 4: Isoliquiritigenin inhibits tube-like structure formation by HUVECs. Cells were fluorescently labeled as described in "Methods" and seeded onto Matrigel in the presence or absence of various concentrations of isoliquiritigenin for $6 \mathrm{~h}$. A set of representative photographs from three independent experiments with similar results is shown.

\section{Isoliquiritigenin suppresses vasculogenesis and corneal neovascularization}

To confirm the anti-angiogenic activity of isoliquiritigenin in vivo, we first used the mouse Matrigel plug model of angiogenesis. Resveratrol was chosen as the positive control because it is often used as an anti-angiogenic agent to treat various angiogenesis-related diseases [17-19]. As shown in Figure 6A, significant blood vessel formation was observed in Matrigel plugs supplemented with fibroblast growth factor (FGF) as compared to the plugs harvested from the control mice. However, microvessel formation induced by FGF was profoundly reduced in the presence of $100 \mu \mathrm{M}$ isoliquiritigenin, as demonstrated by relatively pale color of isoliquiritigenin-supplemented plugs compared to that of vehicletreated plugs, which was more effective than that of resveratrol treatment (Figure 6A). Consistently, isoliquiritigenin potently reduced the hemoglobin content of the plugs (Figure 6B), further confirming that isoliquiritigenin is a potent inhibitor of growth factor-induced angiogenesis in vivo.

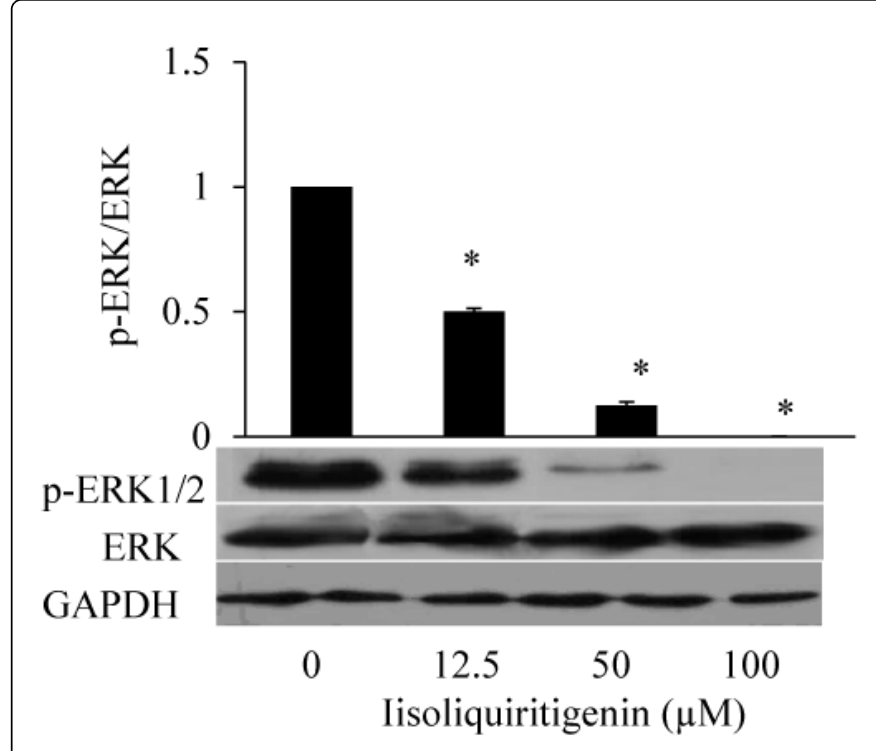

Figure 5: Isoliquiritigenin inhibits ERK1/2 phosphorylation in HUVEs. HUVECs were incubated with vehicle or various concentrations of isoliquiritigenin for $48 \mathrm{~h}$. Western analysis was performed to detect phosphorylated ERK1/2 (p-ERK1/2), which was normalized to total ERK $1 / 2$ from the same sample. Mean \pm SE of normalized densitometry measurements from three separate experiments, along with representative blots, are shown.

To further test the therapeutic potential of isoliquiritigenin in treating corneal neovascularization, we generated a mouse model of corneal neovascularization by alkali burning of the mouse cornea, which causes injury to the corneal surface and subsequent neovascularization, a well-established model for the study of corneal neovascularization. As shown in Figure 7A, topical application of isoliquiritigenin (100 $\mu \mathrm{M}$ in eye drops) to the corneas injured by chemical burn greatly inhibited the development of corneal neovascularization, an effect that was more potent than that of resveratrol. Analysis of sections from H\&E (Figure 7B) and immunohistochemistry staining (Figure 7C) further showed that isoliquiritigenin greatly decreased the numbers of blood/lymphatic vessels in the corneas, which was also more effective than that of resveratrol.

\section{Isoliquiritigenin has no effect on the gene expression of angiogenic factors in cornea}

It is recognized that overexpression of several growth factors in the cornea, including vascular endothelial growth factor (VEGF), basic fibroblast growth factor (bFGF) and transforming growth factor beta1 $(\mathrm{TGF} \beta 1)$, plays pivotal roles in promoting angiogenesis and corneal neovascularization [3,20-22], we then performed RT-PCR to determine whether isoliquiritigenin suppresses neovascularization through suppressing the production of angiogenic factors. The results show that chemical burn drastically increased gene expression of VEGF, bFGF, and TGF $\beta 1$ by $11.8,142.9$ and 3.2 fold, respectively, in the neovascularized corneas from mice. However, neither isoliquiritigenin nor resveratrol significantly affected chemical injuryinduced up regulation of these angiogenic factors (data not shown). 
Citation: Yang L, He W, Qu H, Jia C, Wang Y, et al. (2014) Phytochemical Isoliquiritigenin Inhibits Angiogenesis Ex Vivo and Corneal Neovascularization in Mice. Altern Integr Med 3: 176. doi:10.4172/2327-5162.1000176

Page 6 of 8

A

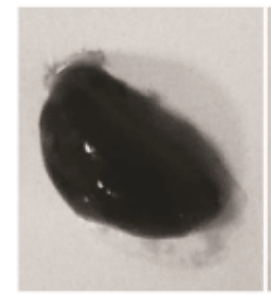

$\mathrm{C}$

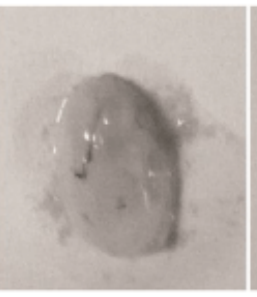

ISL

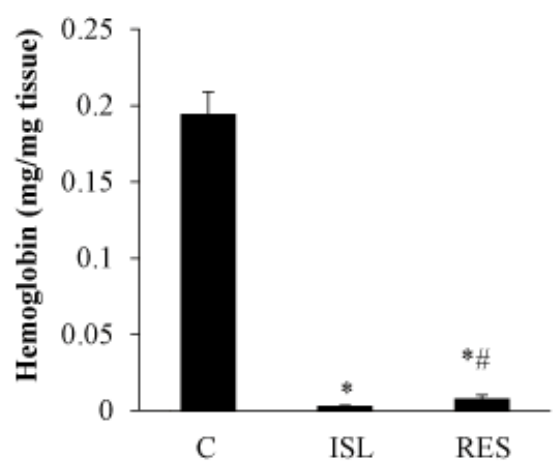

Figure 6: Isoliquiritigenin inhibits angiogenesis in vivo. Matrigels in the presence of basic fibroblast growth factor (bFGF) and heparin with or without $100 \mu \mathrm{M}$ isoliquiritigenin (ISL) or resveratrol (RES) were injected subcutaneously into the abdominal wall of mice. (A) Representative photographs of Matrigel plugs at $10 \mathrm{~d}$ after implantation are shown ( $\mathrm{n}=4$ mice/group). (B) Hemoglobin levels in the Matrigel plugs were quantified and are expressed as mean \pm SE. ${ }^{*}, \mathrm{p}<0.05$ vs. bFGF alone-treated group.

\section{Discussion}

Angiogenesis is a complex process with EC cell proliferation, migration, and tubulogenesis playing an essential role in ultimate new blood vessel formation [15] Corneal angiogenesis causes vascularization of avascular cornea that can lead to the decline of vision and even blindness. The pathogenesis of corneal neovascularization is still not fully understood, but it is stimulated by various growth factors, notably VEGF and bFGF that are secreted in response to hypoxia, chemical burn, chronic inflammation, infection, and trauma [22]. Isoliquiritigenin is a naturally occurring flavonoid that reportedly has an array of biological activities, such as vasorelaxation [23], anti-platelet aggregation [24], anti-inflammation [25], and anti-cancer [26]. While studies examining the effects of this compound on vascular ECs are limited, a recent study showed that isoliquiritigenin suppressed growth factor-stimulated proliferation of ECs [27]. In the present study, we identify that isoliquiritigenin potently inhibits EC proliferation, migration, and the formation of tube-like structure ex vivo. Consistently, we demonstrate that this compound is a strong inhibitor of angiogenesis and corneal neovascularization induced by growth factor and chemical injury in vivo. These data suggest that isoliquiritigenin may have great therapeutic for ocular neovascularization and other disorders associated with angiogenesis.

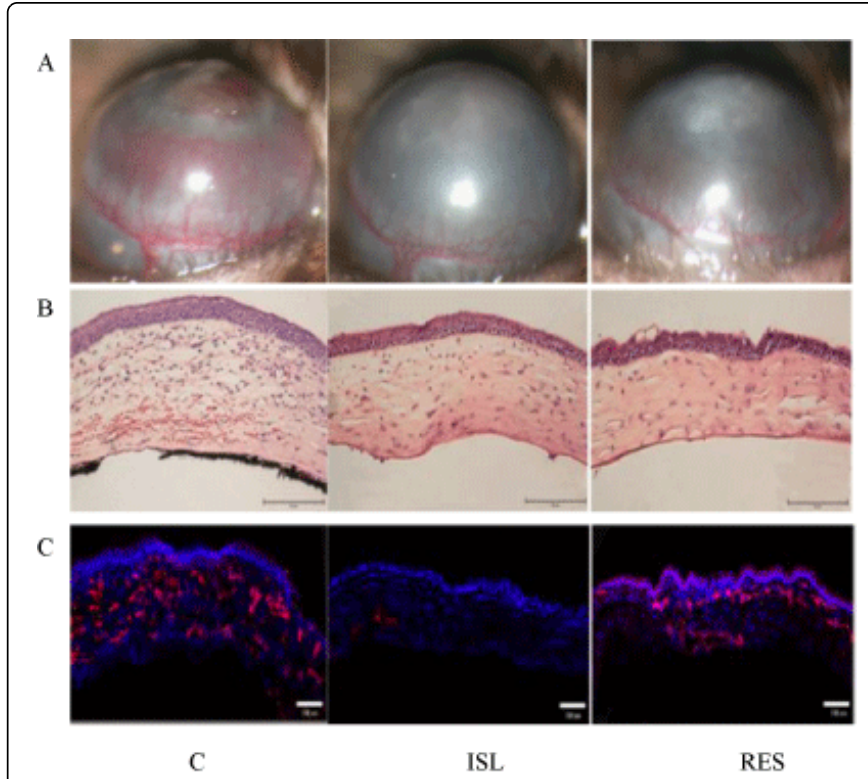

Figure 7: Isoliquiritigenin inhibits corneal neovascularization. Corneal neovascularization in mice was induced as described in the "Methods". Isoliquiritigenin (ISL) or resveratrol (RES) in $100 \mu \mathrm{M}$ solution was then applied topically $(5 \mu \mathrm{L})$ to the eyes 4 times daily for 7 consecutive days. Control eyes were treated with vehicle only. (A) Neovascularization was examined by slit lamp and representative photographs of cornea at day 7 after induction of corneal neovascularization ( $\mathrm{n}=4$ mice/group) are shown. (B) $H \& E$ staining of corneal sections. Corneal neovascularization assessed by CD31 staining. As demonstrated, the area of neovascularized cornea dramatically increased in the control groups but was significantly reduced in mice treated with isoliquiritigenin.

The exact mechanism for isoliquiritigenin to inhibit corneal neovascularization is presently unclear. It is well recognized that the ERK1/2 signaling pathway in vascular ECs plays an important role in cell proliferation. migration, and angiogenesis [28,29]. ERK1/2 are activated in response to a large array of extracellular stimuli, including growth factors [29-31]. In the present study, we show that isoliquiritigenin greatly suppressed ERK1/2 activation, suggesting that its inhibitory action on angiogenesis may be primarily mediated via inhibiting the ERK1/2 signaling pathway. Interestingly, isoliquiritigenin treatment had no effect on ERK1/2 protein expression, suggesting that isoliquiritigenin ablates ERK1/2 activity via a non-genomic mechanism. ERK1/2 is directly activated by MEK, which is activated by its upstream kinase, Raf-1 [32]. However, how isoliquiritigenin interacts with the ERK $1 / 2$ pathway remains to be elucidated.

Accumulating evidence show that several growth factors, particularly VEGF and bFGF, play a critical role in corneal angiogenesis $[3,20,21]$. Indeed, studies showed that these angiogenic factors are over-expressed and increasingly secreted from neovascularized cornea [6]. Accordingly, corneal neovascularization can be reduced by anti-VEGF or anti-bFGF treatment [22,33]. In the present study, our data show that the expression of these angiogenic factors in neovascularized mouse cornea was greatly increased. However, treatment with isoliquiritigenin had no significant effect on these molecules, suggesting that the anti-angiogenesis action of 
isoliquiritigenin is not mediated through suppressing the expression of VEGF and bFGF in the cornea.

Tanshinone IIA, isolated from the root of Salvia miltiorrhiza, is widely used in the prescriptions for treating vascular diseases. While the scientific evidence supporting its beneficial effect on vasculature in humans is not well established, some studies showed that tanshinone IIA protects against vascular inflammation and promotes vasodilatation [34-36]. In addition, tanshinone IIA has been shown to inhibit proliferation and migration of cancer cells [37-39]. In the present study, we demonstrate that tanshinone IIA is a potent agent inhibiting EC proliferation and does-dependently inhibits cell migration, but it is less potent than isoliquiritigenin in inhibiting EC migration, which is essential to angiogenesis. Given this result, we then solely focused on further characterizing the anti-angiogenic action of isoliquiritigenin. However, there is possibility that tanshinone IIA is still a potent anti-angiogenesis agent in vivo, as it may be able to suppress angiogenesis by inhibiting migration of smooth muscle cells, as reported [40].

In summary, by screening herb-derived small molecules, we identified that isoliquiritigenin is a potent inhibitor of angiogenesis and corneal neovascularization in vivo. These effects are ascribed at least in part to the inhibition of proliferation, migration, and tubulogenesis of ECs. These data suggest that isoliquiritigenin may have great therapeutic potential as a low-cost natural compound for neovascularization-associated corneal disorders or other diseases. However, the detailed anti-angiogenic mechanisms of isoliquiritigenin remain to be determined.

\section{References}

1. Iruela-Arispe ML, Dvorak HF (1997) Angiogenesis: a dynamic balance of stimulators and inhibitors. Thromb Haemost 78: 672-677.

2. Isner JM, Asahara T (1999) Angiogenesis and vasculogenesis as therapeutic strategies for postnatal neovascularization. J Clin Invest 103: 1231-1236.

3. Suryawanshi A, Veiga-Parga T, Reddy PB, Rajasagi NK, Rouse BT (2012) IL-17A differentially regulates corneal vascular endothelial growth factor (VEGF)-A and soluble VEGF receptor 1 expression and promotes corneal angiogenesis after herpes simplex virus infection. J Immunol 188: 3434-3446

4. Maruyama K, Ii M, Cursiefen C, Jackson DG, Keino H, et al. (2005) Inflammation-induced lymphangiogenesis in the cornea arises from CD11b-positive macrophages. J Clin Invest 115: 2363-2372.

5. Xue ML, Thakur A, Willcox M (2002) Macrophage inflammatory protein-2 and vascular endothelial growth factor regulate corneal neovascularization induced by infection with Pseudomonas aeruginosa in mice. Immunol Cell Biol 80: 323-327

6. Amano S, Rohan R, Kuroki M, Tolentino M, Adamis AP (1998) Requirement for vascular endothelial growth factor in wound- and inflammation-related corneal neovascularization. Invest Ophthalmol Vis Sci 39: 18-22

7. Sivak JM, Ostriker AC, Woolfenden A, Demirs J, Cepeda R, et al. (2011) Pharmacologic uncoupling of angiogenesis and inflammation during initiation of pathological corneal neovascularization. J Biol Chem 286: 44965-44975.

8. Chen P, Yin H, Wang Y, Mi J, He W, et al. (2010) Multi-gene targeted antiangiogenic therapies for experimental corneal neovascularization. Mol Vis 16: 310-319.

9. Wang Y, Yin H, Chen P, Xie L, Wang Y (2011) Inhibitory effect of canstatin in alkali burn-induced corneal neovascularization. Ophthalmic Res 46: 66-72.
10. Li C, Zhang F, Wang Y (2010) S100A proteins in the pathogenesis of experimental corneal neovascularization. Mol Vis 16: 2225-2235.

11. Maddula S, Davis DK, Maddula S, Burrow MK, Ambati BK (2011) Horizons in therapy for corneal angiogenesis. Ophthalmology 118: 591-599.

12. Koltermann A, Liebl J, Fürst R, Ammer H, Vollmar AM, et al. (2009) Ginkgo biloba extract EGb 761 exerts anti-angiogenic effects via activation of tyrosine phosphatases. J Cell Mol Med 13: 2122-2130.

13. Fan TP, Yeh JC, Leung KW, Yue PY, Wong RN (2006) Angiogenesis: from plants to blood vessels. Trends Pharmacol Sci 27: 297-309.

14. Ishiyama M, Tominaga H, Shiga M, Sasamoto K, Ohkura Y, et al. (1996) A combined assay of cell viability and in vitro cytotoxicity with a highly water-soluble tetrazolium salt, neutral red and crystal violet. Biol Pharm Bull 19: 1518-1520.

15. Liu D, Iruthayanathan M, Homan LL, Wang Y, Yang L, et al. (2008) Dehydroepiandrosterone stimulates endothelial proliferation and angiogenesis through extracellular signal-regulated kinase 1/2-mediated mechanisms. Endocrinology 149: 889-898.

16. McMahon GA, Petitclerc E, Stefansson S, Smith E, Wong MK, et al. (2001) Plasminogen activator inhibitor-1 regulates tumor growth and angiogenesis. J Biol Chem 276: 33964-33968.

17. Khan AA, Dace DS, Ryazanov AG, Kelly J, Apte RS (2010) Resveratrol regulates pathologic angiogenesis by a eukaryotic elongation factor-2 kinase-regulated pathway. Am J Pathol 177: 481-492.

18. Bråkenhielm E, Cao R, Cao Y (2001) Suppression of angiogenesis, tumor growth, and wound healing by resveratrol, a natural compound in red wine and grapes. FASEB J 15: 1798-1800.

19. Athar M, Back JH, Kopelovich L, Bickers DR, Kim AL (2009) Multiple molecular targets of resveratrol: Anti-carcinogenic mechanisms. Arch Biochem Biophys 486: 95-102.

20. Oliveira HB, Sakimoto T, Javier JA, Azar DT, Wiegand SJ, et al. (2010) VEGF Trap(R1R2) suppresses experimental corneal angiogenesis. Eur J Ophthalmol 20: 48-54.

21. Ghanem RC, Han KY, Rojas J, Ozturk O, Kim DJ, et al. (2011) Semaphorin 7A promotes angiogenesis in an experimental corneal neovascularization model. Curr Eye Res 36: 989-996.

22. Shakiba Y, Mansouri K, Arshadi D, Rezaei N (2009) Corneal neovascularization: molecular events and therapeutic options. Recent Pat Inflamm Allergy Drug Discov 3: 221-231.

23. Liu B, Yang J, Wen Q, Li Y (2008) Isoliquiritigenin, a flavonoid from licorice, relaxes guinea-pig tracheal smooth muscle in vitro and in vivo: role of cGMP/PKG pathway. Eur J Pharmacol 587: 257-266.

24. Tawata M, Aida K, Noguchi T, Ozaki Y, Kume S, et al. (1992) Antiplatelet action of isoliquiritigenin, an aldose reductase inhibitor in licorice. Eur J Pharmacol 212: 87-92.

25. Kumar S, Sharma A, Madan B, Singhal V, Ghosh B (2007) Isoliquiritigenin inhibits IkappaB kinase activity and ROS generation to block TNF-alpha induced expression of cell adhesion molecules on human endothelial cells. Biochem Pharmacol 73: 1602-1612

26. Wang KL, Hsia SM, Chan CJ, Chang FY, Huang CY, et al. (2013) Inhibitory effects of isoliquiritigenin on the migration and invasion of human breast cancer cells. Expert Opin Ther Targets 17: 337-349.

27. Cao L, Liu H, Lam DS, Yam GH, Pang CP (2010) In vitro screening for angiostatic potential of herbal chemicals. Invest Ophthalmol Vis Sci 51: 6658-6664.

28. Wu L, Mayo L, Dunbar J, Kessler K, Baerwald M, et al. (2000) Utilization of distinct signaling pathways by receptors for vascular endothelial cell growth factor and other mitogens in the induction of endothelial cell proliferation. Journal of Biological Chemistry 275: 5096-5103

29. Pedram A, Razandi M, Levin ER (1998) Extracellular signal-regulated protein kinase/Jun kinase cross-talk underlies vascular endothelial cell growth factor-induced endothelial cell proliferation. Journal of Biological Chemistry 273: 26722-26728 
Citation: Yang L, He W, Qu H, Jia C, Wang Y, et al. (2014) Phytochemical Isoliquiritigenin Inhibits Angiogenesis Ex Vivo and Corneal Neovascularization in Mice. Altern Integr Med 3: 176. doi:10.4172/2327-5162.1000176

Page 8 of 8

30. Razandi M, Pedram A, Park ST, Levin ER (2003) Proximal events in signaling by plasma membrane estrogen receptors. J Biol Chem 278: 2701-2712.

31. Jo H, Sipos K, Go Y, Law R, Rong J, et al. (1997) Differential effect of shear stress on extracellular signal-regulated kinase and N-terminal Jun kinase in endothelial cells. Gi2- and Gbeta/gamma-dependent signaling pathways. J Biol Chem 272: 1395-1401

32. Simoncini T, Mannella P, Fornari L, Varone G, Caruso A, et al. (2003) Dehydroepiandrosterone modulates endothelial nitric oxide synthesis via direct genomic and nongenomic mechanisms. Endocrinology 144: 3449-3455.

33. Chang JH, Garg NK, Lunde E, Han KY, Jain S, et al. (2012) Corneal neovascularization: an anti-VEGF therapy review. Surv Ophthalmol 57: 415-429.

34. $\mathrm{Xu} \mathrm{W,} \mathrm{Yang} \mathrm{J,} \mathrm{Wu} \mathrm{LM} \mathrm{(2009)} \mathrm{Cardioprotective} \mathrm{effects} \mathrm{of} \mathrm{tanshinone} \mathrm{IIA}$ on myocardial ischemia injury in rats. Pharmazie 64: 332-336.

35. Fan GW, Gao XM, Wang H, Zhu Y, Zhang J, et al. (2009) The antiinflammatory activities of Tanshinone IIA, an active component of TCM, are mediated by estrogen receptor activation and inhibition of iNOS. J Steroid Biochem Mol Biol 113: 275-280.
36. Yin X, Yin Y, Cao FL, Chen YF, Peng Y, et al. (2012) Tanshinone IIA attenuates the inflammatory response and apoptosis after traumatic injury of the spinal cord in adult rats. PLoS One 7: e38381.

37. Wang X, Wei Y, Yuan S, Liu G, Lu Y, et al. (2005) Potential anticancer activity of tanshinone IIA against human breast cancer. Int J Cancer 116: 799-807.

38. Won SH, Lee HJ, Jeong SJ, Lee HJ, Lee EO, et al. (2010) Tanshinone IIA induces mitochondria dependent apoptosis in prostate cancer cells in association with an inhibition of phosphoinositide 3-kinase/AKT pathway. Biol Pharm Bull 33: 1828-1834.

39. Tang C, Xue HL, Huang HB, Wang XG (2010) Tanshinone IIA inhibits constitutive STAT3 activation, suppresses proliferation, and induces apoptosis in rat C6 glioma cells. Neurosci Lett 470: 126-129.

40. Jin UH, Suh SJ, Chang HW, Son JK, Lee SH, et al. (2008) Tanshinone IIA from Salvia miltiorrhiza BUNGE inhibits human aortic smooth muscle cell migration and MMP-9 activity through AKT signaling pathway. J Cell Biochem 104: 15-26 